\title{
Ozone-Induced Dissociation on a Modified Tandem Linear Ion-Trap: Observations of Different Reactivity for Isomeric Lipids
}

\author{
Berwyck L. J. Poad, ${ }^{a}$ Huong T. Pham, ${ }^{a}$ Michael C. Thomas, ${ }^{a}$ \\ Jessica R. Nealon, ${ }^{\mathrm{a}}$ J. Larry Campbell, ${ }^{\mathrm{b}}$ Todd W. Mitchell, ${ }^{\mathrm{c}}$ and \\ Stephen J. Blanksby ${ }^{\mathrm{a}}$ \\ a School of Chemistry, University of Wollongong, Gwynneville, Australia \\ b AB SCIEX, Concord, ON, Canada \\ ' School of Health Sciences, University of Wollongong, Gwynneville, Australia
}

Ozone-induced dissociation (OzID) exploits the gas-phase reaction between mass-selected lipid ions and ozone vapor to determine the position(s) of unsaturation. In this contribution, we describe the modification of a tandem linear ion-trap mass spectrometer specifically for OzID analyses wherein ozone vapor is supplied to the collision cell. This instrumental configuration provides spatial separation between mass-selection, the ozonolysis reaction, and mass-analysis steps in the OzID process and thus delivers significant enhancements in speed and sensitivity (ca. 30-fold). These improvements allow spectra revealing the double-bond position(s) within unsaturated lipids to be acquired within $1 \mathrm{~s}$ : significantly enhancing the utility of OzID in high-throughput lipidomic protocols. The stable ozone concentration afforded by this modified instrument also allows direct comparison of relative reactivity of isomeric lipids and reveals reactivity trends related to (1) double-bond position, (2) substitution position on the glycerol backbone, and (3) stereochemistry. For cis- and trans-isomers, differences were also observed in the branching ratio of product ions arising from the gas-phase ozonolysis reaction, suggesting that relative ion abundances could be exploited as markers for double-bond geometry. Additional activation energy applied to mass-selected lipid ions during injection into the collision cell (with ozone present) was found to yield spectra containing both OzID and classical-CID fragment ions. This combination CID-OzID acquisition on an ostensibly simple monounsaturated phosphatidylcholine within a cow brain lipid extract provided evidence for up to four structurally distinct phospholipids differing in both double-bond position and sn-substitution. (J Am Soc Mass Spectrom 2010, 21, 1989-1999) (c) 2010 American Society for Mass Spectrometry

\section{L} ipids are a diverse group of structurally varied biomolecules that are central to many vital biolular membranses, including the formation cellular membranes, energy storage and even cell signaling [1]. There are a wide range of lipid subclasses with different biochemical roles, including glycerophospholipids (GPLs) that act as primary building blocks of membranes and precursors for intracellular signaling molecules; fatty acids (FAs) and triacylglycerols (TAGs) that are the major source of energy in plants and animals; and sterols that modulate membrane stability and act as biochemical messengers [2]. The specific functions of lipid classes, and indeed individual lipids, are related to their chemical and physical properties that in turn depend on specific molecular features [3]. As such, even small changes in molecular structure can affect the role of a lipid within a living organism. Recent

Address reprint requests to Dr. S. J. Blanksby, Department of Chemistry, University of Wollongong, Northfields Rd., Wollongong, NSW 2522, Australia. E-mail: blanksby@uow.edu.au research has indicated that within living organisms, different lipid isomers play different, and in some cases contrasting, metabolic roles. For example, one study focusing on the effect of conjugated linoleic acid isomers on development of atherosclerosis in ApoE knockout mice revealed that while one isomer (10E,12Z-18:2) had a profound atherogenic effect, an alternate isomer (9Z, 11E-18:2) was anti-atherogenic [4]. While differences in molecular structure arising from double-bond position and/or stereoisomerism (vide infra) can be characterized in simple lipids by traditional chromatographic methods, in complex lipids, such as glycerophospholipids and triacylglycerols, these structural variations are more difficult to identify. The development of analytical technologies that specifically target the identification of double-bond position in unsaturated lipids has recently been reviewed [4].

Further structural diversity in glycerolipids can arise from the relative position of fatty acid substituents on the glycerol backbone. Such sn-positional isomers are difficult to distinguish by mass spectrometry and thus 
structures are often reported as a single isomer based on assumptions about commonly occurring motifs (e.g., in glycerophospholipids unsaturated radyls are often assigned to the $s n-2$ position). Such unequivocal assignments are made despite the fact that numerous studies employing either selective enzymes or chromatographic methods have shown that both glycerophospholipids and triacylglycerols often appear in biology as mixtures of sn-positional isomers [5-9]. Despite the recent interest in the involvement of trans-fatty acids in increasing the risk of several chronic human diseases, particularly coronary heart disease [10, 11], there has been relatively little attention paid to the possibility of stereoisomerism in complex lipids. This arises because differentiating stereoisomers represents a fundamental challenge for mass spectrometry.

Shotgun lipidomics refers to contemporary mass spectrometric methods that can rapidly identify and quantify hundreds of individual lipid molecules within a crude extract [12-14]. These methods are based on the combination of electrospray ionization with tandem mass spectrometry (ESI-MS/MS), which provides detailed structural information for individual lipids based on their mass-to-charge ratio and characteristic fragment ions. For example, the head-group class and the number of carbons and number of double bonds in each of the fatty acyl chains can be readily assigned for glycerophospholipids from ESI-MS/MS data [15, 16]. Furthermore, compared with internal standards of the same lipid class, ion abundances can be used to quantify individual lipids within a sample [17]. Shotgun lipidomics represents a significant evolution in lipid analysis and, through harnessing the mass-selection capabilities of tandem mass spectrometry, is providing new insights into the biochemical and physiological role of molecular lipids and their association with disease [18].

Despite these advances, ascertaining a complete structural characterization of molecular lipids by mass spectrometry alone presents significant challenges. Contemporary lipidomic analyses are overwhelmingly conducted on triple quadrupole mass spectrometers and thus collision-induced dissociation (CID) is conducted in the low-energy range with collision energies less than $100 \mathrm{eV}$. CID spectra acquired under these conditions, in either positive or negative ion mode, can provide an indication on the relative position of the acyl chains on the glycerol backbone (often referred to as $s n$-position) from the abundance of product ions arising from dissociation at the fatty acyl moieties $[6,19,20]$. In contrast, low-energy CID spectra fail to provide any distinctive spectral features indicative of either (1) the position of carbon-carbon double-bond(s) or (2) the stereochemistry of the double bonds (cis or trans). Publications from our group and others have contrasted the CID mass spectra of double-bond variants of complex molecular lipids, demonstrating the inability of this approach to differentiate such isomers [21, 22]. As such, a conventional CID spectrum of a mass-selected lipid provides little information to alert the analyst to the presence of isomeric variations in lipids (i.e., Is a mass-selected ionized lipid one species or, in fact, a mixture of several structurally distinct compounds?).

There are several approaches available to differentiate and identify double-bond isomers in intact molecular lipids [4]. Of these approaches, only three operate on mass-selected ions in tandem mass spectrometers, namely (1) high-energy CID; (2) multi-stage fragmentation approaches using highly metallated lipids; and (3) ozone-induced dissociation. Extensive work on sector mass spectrometers over many years has demonstrated that $\mathrm{keV}$ collision energies induce dissociation of carbon-carbon bonds (by so-called charge-remote fragmentation) in ionized lipids and thereby allow the assignment of double-bond position and, in some instances, stereochemistry [23, 24]. Indeed for triacylglycerols, Gross and coworkers demonstrated that the combination of charge-directed and charge-remote fragmentation in the high-energy CID spectra of metallated-lipids could be used to assign both sn- and double-bond-position [25]. While sector mass spectrometers are now no longer widely available, the recent commercialization of tandem time-of-flight (TOF-TOF) mass spectrometers that are also capable of collision energies in the $\mathrm{keV}$ range are showing promise for reinvigorating this approach to more comprehensive lipid structure elucidation [26]. An alternative approach using low-energy CID has been demonstrated by Hsu and Turk, and employs sequential fragmentation of metallated lipids on ion-trap mass spectrometers [27, 28]. In this approach, lithiated phospholipids and triacylglycerols are subject to primary fragmentation $\left(\mathrm{MS}^{2}\right)$ to produce conventional charge-induced fragmentation, with subsequent low-energy dissociation of these product ions (MS ${ }^{n}$, where $n>2$ ) giving rise to charge-remote processes that reveal the position of the double bonds. While both the high-energy and multi-stage CID approaches can be used to provide a near complete structural proof for a complex lipid, both give rise to complicated spectra thereby making analysis of isomeric mixtures difficult without prior separation (i.e., chromatography). Furthermore, neither approach can be readily employed on a triple quadrupole mass spectrometer.

We have previously developed a technique that exploits the gas-phase reaction between ozone and carbon-carbon double bonds within ionized lipids that have been mass-selected within a linear ion-trap mass spectrometer. This approach, termed ozone-induced dissociation (OzID), yields predictable fragment ions revealing the double-bond position within the fatty acyl chains of intact molecular lipids [29-31]. In this contribution, we present a significant extension of the previous experimental design through the modification of a tandem linear ion-trap mass spectrometer [32] for conducting OzID analyses. Given that triple quadrupole instruments are the workhorses of contemporary lipidomic analysis, the present geometry, (which also op- 
erates as a triple quadrupole mass spectrometer), seamlessly integrates with already established workflows. This configuration also differs from our previous work by facilitating the continuous generation and stable on-line delivery of high concentration ozone to the mass spectrometer. The stability of ozone levels within the instrument allows, for the first time, direct observation of differences in reactivity between isomeric unsaturated lipids. Herein, it is revealed that not only do the position and stereochemistry of double-bond influence the ozonolysis reaction rate, but also the position of unsaturated acyl chain on the glycerol backbone (snposition) has a noticeable effect.

\section{Experimental}

\section{Materials and Sample Preparation}

Synthetic phospholipid standards were purchased from Avanti Polar Lipids, Inc. (Alabaster, AL, USA), while the fatty acids were obtained from NU-CHEK PREP Inc. (Elysian, MN, USA). All lipid standards were used without further purification. HPLC grade methanol and AR grade sodium acetate were purchased from APS Chemicals (Sydney, NSW, Australia). High-purity compressed oxygen was purchased from BOC gases (Cringila, NSW, Australia). Standard solutions of lipids for electrospray ionization were prepared in methanol at a concentration of $\sim 10 \mu \mathrm{M}$. [M $+\mathrm{Na}]^{+}$adduct ions have previously been demonstrated to react more efficiently with ozone than their $[\mathrm{M}+\mathrm{H}]^{+}$analogues [29]. To facilitate the formation of sodium adduct ions, sodium acetate was added to the lipid solutions to a final concentration of 50-100 $\mu \mathrm{M}$. Pure Spanish olive oil (Always Fresh, Scoresby, VIC, Australia) was diluted to $\sim 12 \mu \mathrm{M}$ in methanol with $100 \mu \mathrm{M}$ sodium acetate for mass spectrometric analysis. Cattle (Bos taurus L., herein referred to as cow) brain was collected from the Wollondilly Abattoir (Picton, NSW, Australia) immediately following the death of each animal. Organs were transported on ice then stored at $-80{ }^{\circ} \mathrm{C}$ and lipids were extracted as previously described [33]. Cow brain extracts were diluted to a final concentration of $\sim 40 \mu \mathrm{M}$ with the addition of methanol:chloroform (2:1 vol:vol) and aqueous sodium acetate was added before being infused into the mass spectrometer.

\section{Lipid Nomenclature}

Where possible, this study adopts the nomenclature and abbreviation conventions of Fahy et al. [13, 34], where the position of the double-bond in any carbon chain is indicated by the number of bonds from the glycerol end of the chain and immediately precedes the $E$ or $Z$, which defines the stereochemistry about the alkene [e.g., 1-hexadecanoyl-2-(9Z-octadecenoyl)-snglycero-3-phosphocholine is represented as PC(16:0/ 9Z-18:1)]. Conversely, the " $n$ " nomenclature refers to the position of the double-bond as counted from the methyl end of the carbon chain [e.g., PC(16:0/9Z-18:1) includes an n-9 double-bond]. While this is not standard chemical nomenclature, it remains a commonly used classification of fatty acids. In this paper, the " $n$ " nomenclature is instructive because the values of neutral loss due to ozone-induced dissociation are common to all lipids with double bonds at the same position in the chain with respect to the methyl terminus.

\section{Instrumentation}

The experiments were conducted within a modified tandem linear ion-trap mass spectrometer (2000 QTRAP; AB SCIEX, Concord, ON, Canada) [32]. A schematic of the instrument, including the reactant gas introduction system, is shown in Figure 1. Dilute samples in methanol or methanol/chloroform (vide supra) were introduced to the ESI source at a flow rate of 5 $\mu \mathrm{L} \cdot \min ^{-1}$. Typical source conditions consisted of an ion spray voltage of $5 \mathrm{kV}$, source temperature of $80^{\circ} \mathrm{C}$ and declustering potential of $60 \mathrm{~V}$. Ions of interest were mass-selected by the primary quadrupole (Q1) using an isolation width of $3 \mathrm{Da}$, then trapped within the LINAC collision cell (q2) [35] and allowed to react in the presence of $\mathrm{O}_{3}, \mathrm{O}_{2}$ and $\mathrm{N}_{2}$. Fragmentation products arising from either collisional or chemical activation were then analyzed by mass-dependent axial ejection [36] from a linear quadrupole ion-trap (Q3). The OzID scan method is based on a modified time delayed fragmentation (TDF) experiment [37] incorporated in the instrument control software (Analyst 1.5; $\mathrm{AB}$ SCIEX). Once the ions enter $\mathrm{q} 2$, which contains $\sim 10^{-3}$ Torr of a mixture of $\mathrm{O}_{3}, \mathrm{O}_{2}$, and $\mathrm{N}_{2}$, the potentials on the inter-quadrupole focusing lenses, IQ2 and IQ3, are raised to $100 \mathrm{~V}$ to trap the ions for between $10 \mathrm{~ms}$ and $25 \mathrm{~s}$. After the reaction period, the potential on IQ3 was lowered, allowing the products to enter and accumulate in Q3. Ions can also be subjected to a small acceleration potential (typically $15 \mathrm{~V}$ ) between q2 and Q3. This may be used to provide additional activation of the $[\mathrm{M}+\mathrm{Na}+$ $\left.\mathrm{O}_{3}\right]^{+}$ozone adduct and thus increase the yield of ozone-induced fragment ions. After a 50-ms delay, the ions were scanned out of Q3 and a mass spectrum

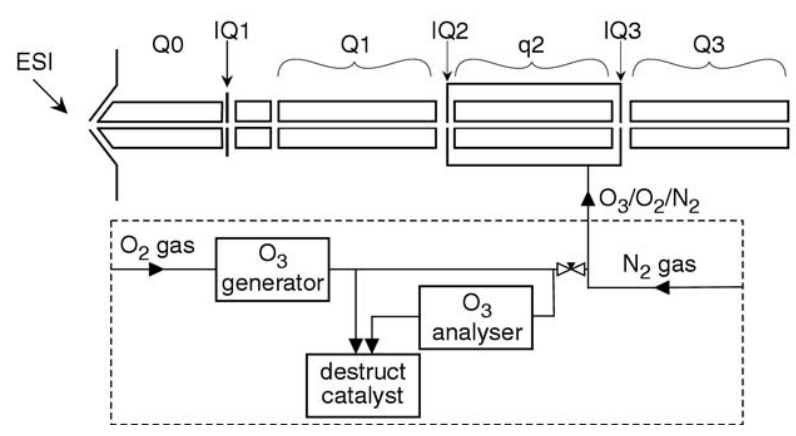

Figure 1. Schematic of the tandem linear ion-trap mass spectrometer (2000 QTRAP) modified to allow the introduction of ozone vapor into the collision cell (q2). 
acquired. Unless otherwise stated, all spectra presented are composed of an average of 50 scans, each employing a trapping time in $\mathrm{q} 2$ of $2 \mathrm{~s}$ and an acceleration voltage of $15 \mathrm{~V}$, representing a total acquisition time of $2 \mathrm{~min}$.

\section{Ozone Generation}

Ozone was produced by a high-concentration ozone generator (Titan 30; Absolute Systems, Edmonton, AB, Canada). A schematic overview of the ozone production and delivery system is shown in Figure 1. Highpurity oxygen was introduced into the generator at a pressure of $20 \mathrm{psi}$ and the generator's corona discharge current set to 40 (arbitrary units). To ensure stable ozone concentration, the generator was run for at least $30 \mathrm{~min}$ before data collection. An inline ozone analyzer (Mini HiCon; InUSA Inc., Norwood, MA, USA) was used to measure the ozone content of the oxygen/ozone gas mixture being introduced to the instrument. Typical ozone content was $140-160 \mathrm{~g} \cdot \mathrm{m}^{-3}$ (ca. $11 \%-12 \% \mathrm{O}_{3}$ in $\mathrm{O}_{2}$ by mass) at a flow rate of $300-400 \mathrm{~mL} \cdot \mathrm{min}^{-1}$. The ozone/oxygen gas mixture was injected into the main nitrogen CID gas line through a T-junction, while excess ozone was destroyed by commercial ozone destruct units (InUSA Inc.). Since ozone is a corrosive gas, all tubing used to construct the gas manifold and ozone delivery system was either 316 stainless steel or Teflon. The gas flow into the instrument was adjusted by a variable leak valve (VSE Vacuum, Lustenau, Austria) such that the pressure reading from the ionization gauge was $1.8 \times 10^{-5}$ Torr. It should be noted that OzID spectra acquired on different days may have been measured with slightly different ozone concentrations in $\mathrm{q} 2$ and thus ion abundances are not directly comparable. Where relative rates of reaction are compared (vide infra), spectra were acquired consecutively, and these measurements were bracketed by observations of a well-characterized compound to check for drift in ozone concentration.

\section{Safety Warning}

Ozone is a toxic gas with an Immediately Dangerous to Life and Health Level proscribed by the Centers for Disease Control and Prevention of only 5 ppm [38]. As such, leak detectors (EcoSensor EZ-1X; Ozone Solutions Inc., Sioux Center, IA, USA) were fitted to the systems and continuously monitored for ozone leaks in the production and delivery system.

\section{Results and Discussion}

\section{Optimization of Instrumental Conditions for OzID}

Figure 2 shows OzID spectra of the sodium adduct ion of trioleoylglycerol obtained from electrospray ionization of a methanolic dilution of olive oil using the modified tandem linear ion-trap. The spectra arise from: (1) mass-selection of $[\mathrm{M}+\mathrm{Na}]^{+}$ions at $m / z 907.8$

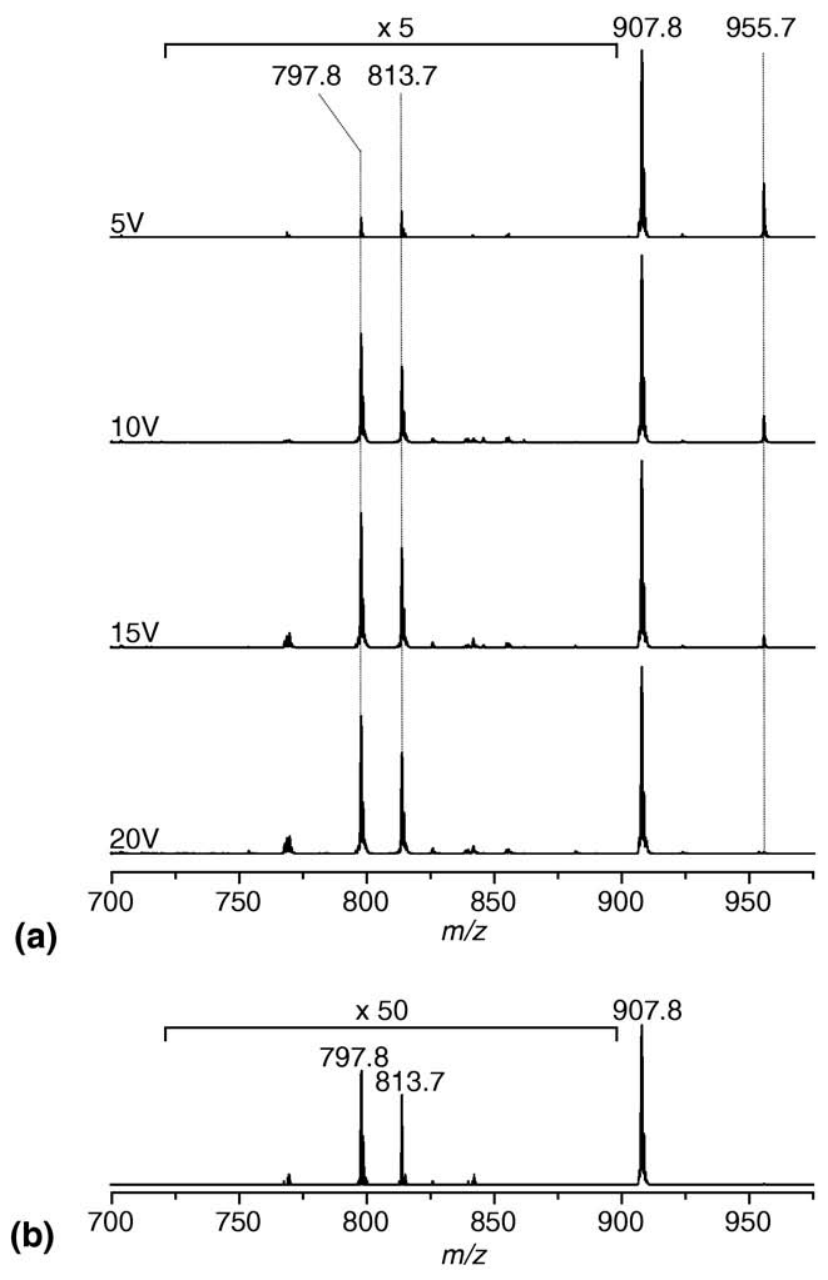

Figure 2. (a) OzID mass spectra obtained at various time delayed fragmentation voltages for [TAG(18:1/18:1/18:1) + Na] ${ }^{+}(\mathrm{m} / \mathrm{z}$ 907.8) mass-selected from an olive oil extract. As the fragmentation voltage is increased, a decrease is noted in the abundance of the ozone adduct peak at $\mathrm{m} / \mathrm{z} 955.7$ accompanied by an increase in the abundance of characteristic OzID fragments at $\mathrm{m} / \mathrm{z} 797.8$ and 813.7 (aldehyde and Criegee ions, respectively). (b) OzID spectrum of the same triacylglycerol using a trapping time of $100 \mathrm{~ms}$ and a supplemental voltage of $15 \mathrm{~V}$. The total acquisition time for this spectrum was $1 \mathrm{~s}$. Despite low overall signal intensity, the sensitivity of the technique is such that the characteristic OzID fragment peaks are still apparent at a good signal-to-noise ratio.

by Q1, (2) trapping of the ions in q2 in the presence of ozone vapor for $2 \mathrm{~s}$, and (3) a second stage of trapping and mass analysis in Q3 (see Figure 1). This sequence gives rise to an $\left[\mathrm{M}+\mathrm{Na}+\mathrm{O}_{3}\right]^{+}$adduct ion at $\mathrm{m} / \mathrm{z} 955.7$ and chemically induced fragment ions at $m / z 797.8$ and 813.7 that correspond to neutral losses of -110 and -94 $\mathrm{Da}$, respectively. These characteristic OzID fragments identify the position of the double bonds as $n-9$ in each acyl chain $[29,31]$. Scheme 1 outlines a possible mechanism for the gas-phase reaction of (1) [TAG(9Z-18:1/ 9Z-18:1/9Z-18:1) $+\mathrm{Na}^{+}$with ozone initiated by the formation of (2) a primary ozonide that may undergo either a unimolecular rearrangement to form (3) a secondary ozonide, or fragmentation to form (4) an aldehyde ion, or (5) a carbonyl oxide intermediate. This 


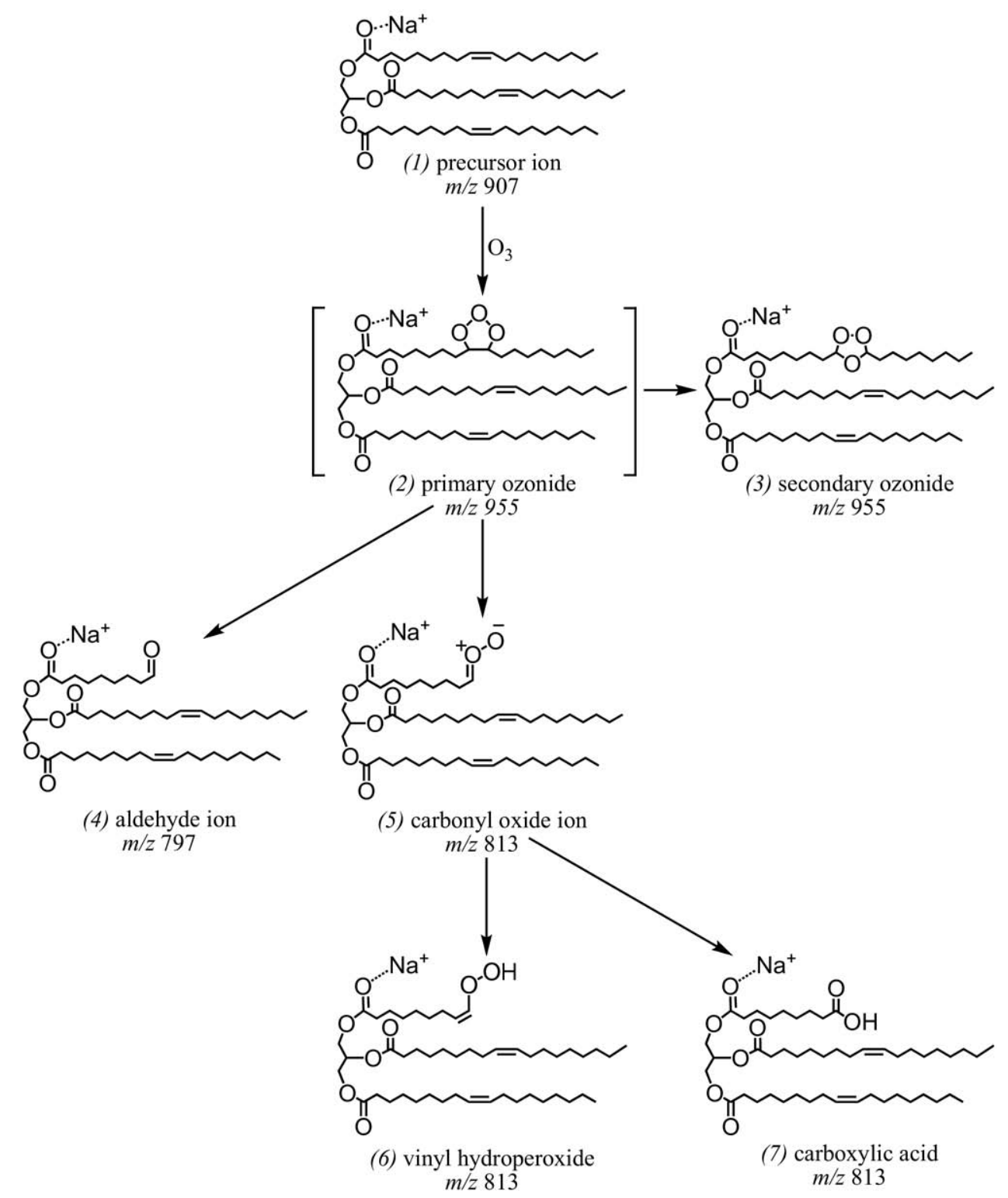

Scheme 1. Proposed mechanism for OzID fragmentation of the triacylgyceride ion [TAG(9Z-18:1/ 9Z-18:1/9Z-18:1) + Na] $]^{+}$.

last fragment is a highly reactive species originally proposed by Criegee [39] and recently observed for the first time by Taatjes et al. [40]. Given the reactive nature of this intermediate, further rearrangement to (6) a carboxylic acid, (7) a vinyl peroxide or even other isobaric structures is probable; these reaction products are thus collectively referred to as "Criegee" ions.

The implementation of OzID on a tandem linear ion-trap instrument presents some key advantages. In previous OzID experiments, ozone (12\% vol/vol in dioxygen) was mixed with helium buffer gas and flowed directly into the mass analysis stage of a linear ion-trap mass spectrometer. Even in moderate concentrations, the presence of heavy molecules in the buffer gas caused a broadening of the peaks and a slight shift in the mass calibration of the instrument that served to limit the partial pressure of ozone to ca. $5 \times 10^{9}$ molecules.cm ${ }^{-3}[29,41]$. On the tandem linear ion-trap mass spectrometer in the present study (see Figure 1), the spatial separation of the ion-molecule reaction region (q2) from the mass analysis (Q3) allowed for a higher concentration of ozone vapor to be used without impacting the resolution or the mass accuracy. In the present configuration, the typical concentration of ozone used in these experiments was estimated to be ca. $5 \times 10^{10}$ molecules. $\mathrm{cm}^{-3}$ using the known second-order rate constant for the reaction $\mathrm{I}^{-}+\mathrm{O}_{3} \rightarrow \mathrm{IO}_{3}{ }^{-}$[42]. Furthermore, this particular instrument is calibrated to use a heavier gas (dinitrogen) as both collision and buffer gases in $\mathrm{q} 2$ and Q3, respectively. Thus, the presence of ozone and dioxygen vapor is less disruptive to mass analysis. Repeat measurements of the reaction 
of $\mathrm{I}^{-}$with $\mathrm{O}_{3}$ over several hours demonstrated the ability to stabilize the ozone concentration over extended periods following an initial period of passivation (see Supplementary Material, which can be found in the electronic version of this article, Figure S7).

The formation of structurally informative OzID ions was further enhanced by the application of a supplemental voltage applied between $\mathrm{q} 2$ and Q3 as a part of the OzID protocol. OzID spectra obtained using supplemental voltages ranging from 5 to $20 \mathrm{~V}$ are shown in Figure 2a and reveal a decrease in the abundance of the $\left[\mathrm{M}+\mathrm{Na}+\mathrm{O}_{3}\right]^{+}$ozonide at $\mathrm{m} / \mathrm{z} 955.7$ and a concomitant increase in the abundance of the structurally informative fragment ions at $\mathrm{m} / \mathrm{z} 797.8$ and 813.7.

Using a combination of high ozone concentration and supplemental activation between q2 and Q3, it was possible to reduce the reaction time for the OzID experiment. Figure $2 b$ shows an OzID spectrum obtained from the same triacylglycerol using a reaction time of $100 \mathrm{~ms}$ and a supplemental activation voltage of $15 \mathrm{~V}$. The spectrum represents an average of only 6 scans, required a total acquisition time of less than $1 \mathrm{~s}$ and consumed only $4 \mathrm{pmol}$ of lipid. Even though the fragment region of the spectrum has been magnified $50 \times$, the signal-to-noise ratio remains excellent. In an effort to assess the improvement in sensitivity achieved here, it is informative to compare the spectrum in Figure $2 \mathrm{~b}$ with that previously obtained on a singlestage linear ion-trap mass spectrometer. In 2008, we published an OzID spectrum of the $[\mathrm{M}+\mathrm{Na}]^{+}$ion of trioleoylglycerol obtained from an olive oil extract measured on such a single-stage instrument (see Figure $7 \mathrm{~d}$ in reference [29]). What required a 30-s acquisition time on the original instrument now requires only a 1-s acquisition time (Figure $2 b$ ) to obtain an OzID spectrum with similar signal-to-noise ratio (at an identical flowrate of $5 \mu \mathrm{L} \cdot \mathrm{min}^{-1}$ ). Notwithstanding that the earlier study used a higher concentration of the lipid extract (ca. $45 \mu \mathrm{M}$ compared to $12 \mu \mathrm{M}$ used here) and required a significantly greater degree of magnification of the spectrum $(3000 \times$ versus $50 \times$ magnification used in Figure $2 b$ ), this provides a conservative estimate of 30-fold improvement in sensitivity in the present configuration. This enhancement in speed (i.e., reduction in acquisition time from 30 to $1 \mathrm{~s}$ ) and consequent improvement in sensitivity of OzID on the tandem linear ion-trap mass spectrometer suggests it could now be integrated into high-throughput "shotgun" lipidomic workflows or on-line LC-MS analyses.

\section{Comparison of Relative Reactivity of Isomeric Lipids}

The rate constants for the gas-phase reactions of ozone with a wide range of neutral olefins have previously been measured because of the importance of such processes to tropospheric processing of hydrocarbons $[43,44]$. In contrast, no information on the relative rates of reaction of ionized olefins with ozone has thus far been reported. The continuous introduction of ozone into the modified tandem linear ion-trap mass spectrometer described here delivers a stable concentration of ozone to q2 and thus allows for the comparison of reaction rates for the first time. The stability of the ozone concentration was checked by comparison of fragment ion abundances in repeat measurements of an OzID spectrum of the well-characterized trioleoylglycerol (reaction time $1 \mathrm{~s}$ and supplemental voltage $15 \mathrm{~V}$ ). Reproducible fragment ion abundances in the resulting spectra provided confirmation of a stable ozone concentration over periods of several hours.

Electrospray ionization of methanolic solutions of fatty acids in the presence of sodium acetate under the standard conditions used in this study gave rise to [M $\mathrm{H}+2 \mathrm{Na}]^{+}$cations as the most abundant ionized lipids. The OzID spectra of the $[\mathrm{M}-\mathrm{H}+2 \mathrm{Na}]^{+}$ions of two isomeric monounsaturated 18-carbon fatty acids, oleic acid (9Z-18:1) and petroselinic acid (6Z-18:1), were recorded consecutively with a common reaction time of $500 \mathrm{~ms}$ (Figure 3a and b, respectively). The pairs of product ions observed in each of these spectra are characteristic of the expected $n-9(-94$ and $-110 \mathrm{Da})$ and $n-12(-136$ and $-152 \mathrm{Da})$ double-bond positions in oleic and petroselinic acid, respectively. Since both spectra were acquired under the same experimental conditions, this allows comment to be made on the relative reactivity of the two 18:1 isomers. For oleic acid, the aldehyde ion at $\mathrm{m} / \mathrm{z} 217.1$ is the base peak in the spectrum, whereas for petroselinic acid, the intensity of the equivalent fragment at $\mathrm{m} / \mathrm{z} 175.0$ is only ca. $70 \%$ of the abundance of the unreacted precursor ion. By integration and normalization of the peak areas, one can infer that for the $[\mathrm{M}-\mathrm{H}+2 \mathrm{Na}]^{+}$ions the $n-9$ fatty

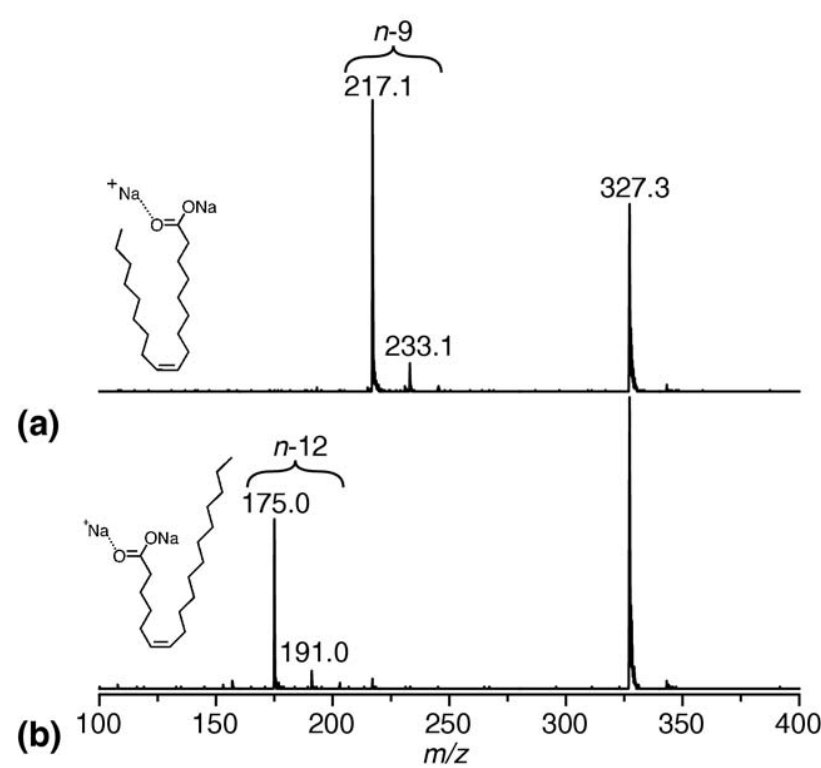

Figure 3. Ozone-induced dissociation spectra for $[\mathrm{M}-\mathrm{H}+$ $2 \mathrm{Na}]^{+}$adduct ions of (a) oleic acid (9Z-18:1), and (b) petroselinic acid (6Z-18:1). All spectra were acquired using a 500-ms trap time. 
acid reacts ca. 5-times faster with ozone than an analogous $n$-12 lipid.

A similar trend in reactivity appears when these isomeric fatty acids are incorporated into phospholipids. Figure 4 shows a comparison of the OzID spectra obtained for the lipids [PC(9Z-18:1/9Z-18:1) $+\mathrm{Na}^{+}$ and [PC(6Z-18:1/6Z-18:1) $+\mathrm{Na}^{+}$. The neutral losses observed in these spectra identify $n-9(-94$ and -110 $\mathrm{Da})$ and $n-12$ ( -136 and $-152 \mathrm{Da})$ double-bond positions. Both spectra in Figure 4 were acquired consecutively with a common reaction time (2 s) and thus, a comparison of the abundance of the product ions suggests that the $n-9$ phospholipid is some 2.5-times more reactive than its $n-12$ isomer. Interestingly, a peak at $m / z$ 749.7 is observed in both OzID spectra and can confidently be assigned to the CID neutral loss of trimethylamine (-59 Da) from the phosphocholine head-group $[20,45]$. This fragmentation arises from the activation energy applied to enhance dissociation of the ozone adducts and suggests that, with careful control of the conditions, it may be possible to obtain both CID and OzID fragments in a single spectrum, thus maximizing spectral information (vide infra).

The OzID spectra for the $[\mathrm{M}+\mathrm{Na}]^{+}$ions formed from electrospray ionization of the stereoisomeric phosphatidylglycerols, PG(9Z-18:1/9Z-18:1) and PG(9E-18: 1/9E-18:1), are shown in Figure $5 a$ and $b$, respectively. The spectra were recorded consecutively using a common OzID reaction time (2 s). Since the isomers are $n-9$ lipids, both spectra exhibit product ions at $m / z 687.5$ and 703.5 corresponding to the characteristic neutral loss of -110 and $-94 \mathrm{Da}$ as previously discussed. Despite the similarities in these spectra, the intensities of the peaks attributable to the ozonolysis products are clearly different, i.e., [PG(9E-18:1/9E-18:1) $+\mathrm{Na}^{+}$is nearly twice as reactive with ozone as [PG(9Z-18:1/9Z-18:1) $+\mathrm{Na}]^{+}$. This trend is in good agreement with previous gas-

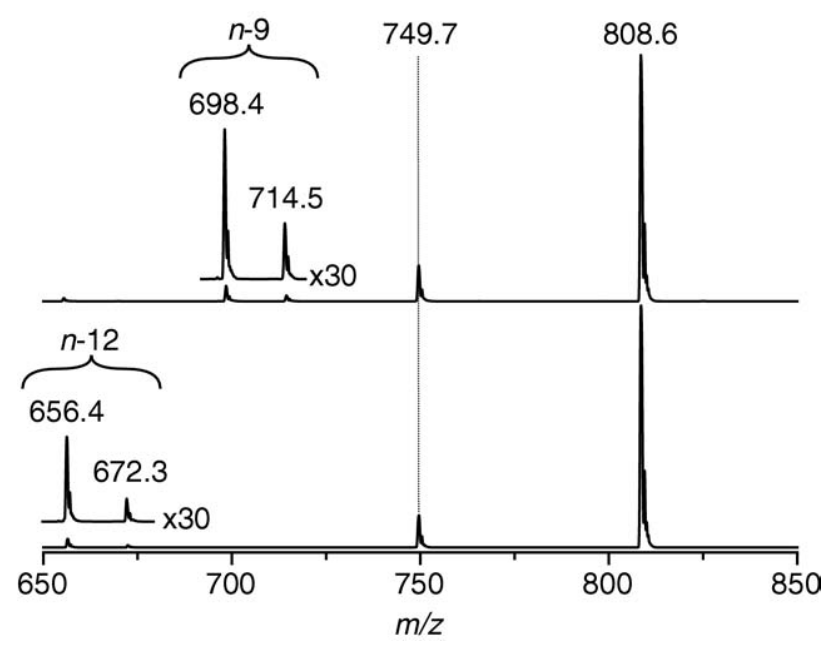

Figure 4. Two OzID spectra illustrating the different reactivity of the $[\mathrm{M}+\mathrm{Na}]^{+}$adduct ions of the phosphatidylcholine regioisomers PC(9Z-18:1/9Z-18:1) (top trace) and PC(6Z-18:1/6Z-18:1) (bottom trace).

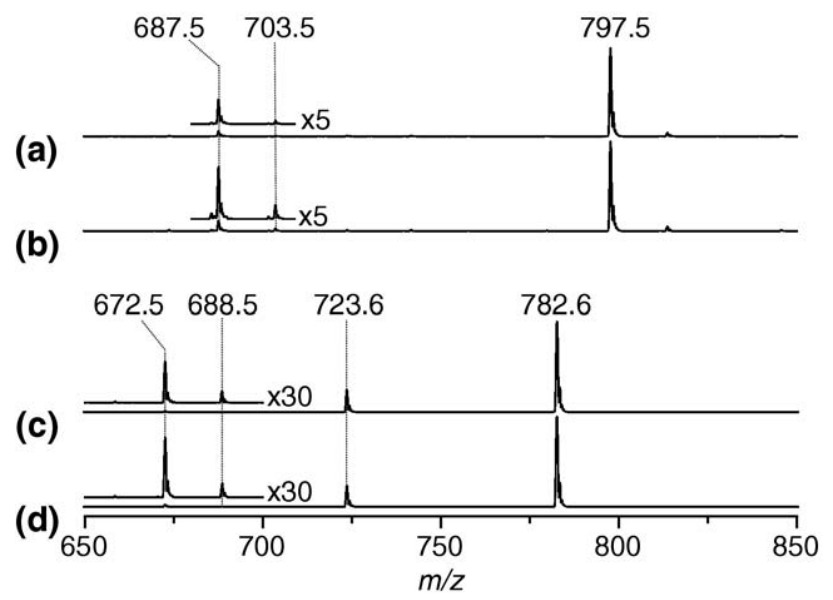

Figure 5. Ozone-induced dissociation mass spectra of the $[\mathrm{M}+$ $\mathrm{Na}]^{+}$adduct ions formed from; the cis- and trans-stereoisomers (a) PG(9Z-18:1/9Z-18:1) and (b) PG(9E-18:1/9E-18:1); and the regioisomers (c) PC(9Z-18:1/16:0) and (d) PC(16:0/9Z-18:1). Each pair of spectra were recorded under identical reaction conditions.

phase kinetic measurements of ozone reacting with neutral cis- and trans-alkenes. Greene and Atkinson found a factor of two difference between the reactivity of cis-2-butene and trans-2-butene towards ozone, with the latter being the more reactive species [46]. Analogous measurements of the phosphatidylcholine isomers [PC(9Z-18:1/9Z-18:1) + Na] ${ }^{+}$and [PC(9E-18:1/9E-18:1) + $\mathrm{Na}]^{+}$and the fatty acid isomers $[9 \mathrm{Z}-18: 1-\mathrm{H}+2 \mathrm{Na}]^{+}$ and $[9 E-18: 1-\mathrm{H}+2 \mathrm{Na}]^{+}$in the present work, yield relative rates of 2.5-times and 1.3-times, respectively, in favor of the trans isomer (see Figures S2 and S3 in Supporting Material).

Comparing the OzID spectra of the geometric isomers in Figure $5 a$ and $b$, it is also interesting to note that the relative abundance of the two product ions at $\mathrm{m} / \mathrm{z}$ 687.5 and 703.5 differs significantly between the cis- and trans-isomers (abundance ratios of $\mathrm{A}_{687} / \mathrm{A}_{703} \sim 16.7$ and 4.1 , respectively). This is consistent with detailed theoretical studies that show product branching in ozonolysis reactions are sensitive to the structure of the primary ozonide [47] that in turn will be influenced by the double-bond geometry (i.e., cis or trans). This trend can also be observed in the OzID spectra of isomeric phosphatidylcholine and fatty acid isomers (see Supplementary Material Figures S2 and S3). The differences observed between stereoisomers in both (1) the overall abundance of OzID fragment ions (i.e., the extent of the reaction) and (2) the relative abundance of the two product ions (i.e., product branching ratio) suggest that OzID spectra could be used to assign double-bond stereochemistry. It should be stressed however, that unlike the use of OzID for identification of double-bond position that arises from unique product ions, differentiation of double-bond stereoisomers relies on different abundances of identical ions. As such, any determination would have to be made under carefully controlled conditions (such as those used here) and with reference to appropriate standards. 
Previous studies on the CID behavior of ionized phospholipids (both in positive- and negative-ion mode) have found that while the same product ions are typically observed from sn-positional isomers, the relative abundance of these ions is dependent on the position of substitution $[6,20]$. For example, Hsu and Turk have shown that for CID of lithiated phosphatidylcholines, the abundance of the $[\mathrm{M}+$ Li-59$\left.\mathrm{R}^{1} \mathrm{CO}_{2} \mathrm{H}\right]^{+}$product ion is greater than the corresponding $\left[\mathrm{M}+\mathrm{Li}-59-\mathrm{R}^{2} \mathrm{CO}_{2} \mathrm{H}\right]^{+}$ion, where $\mathrm{R}^{1} \mathrm{CO}_{2} \mathrm{H}$ and $\mathrm{R}^{2} \mathrm{CO}_{2} \mathrm{H}$ are the fatty acids substituted at the $s n-1$ and $s n-2$ positions, respectively [20]. Indeed this trend in the relative product ion abundances was also observed in the conventional CID spectra of the $[\mathrm{M}+\mathrm{Na}]^{+}$ions of the isomeric phospholipids, PC(16:0/9Z-18:1) and PC(9Z-18:1/16:0) obtained in this study (see Supporting Material Figure S5). The distinctive unimolecular chemistry of these isomers suggests that perhaps the bimolecular processes might also be influenced by $s n$-position. In an effort to establish the effect of $s n$ position on the gas-phase reactivity of ionized glycerophospholipids, the OzID spectra of the isomeric phosphatidylcholine standards, PC(16:0/9Z-18:1) and PC(9Z-18:1/16:0) were measured in consecutive experiments (Figure $5 c$ and d). In these experiments, the $[\mathrm{M}+$ $\mathrm{Na}]^{+}$adduct ions for each isomer was isolated in Q1 and trapped in $\mathrm{q} 2$ in the presence of ozone for $2 \mathrm{~s}$. The spectra reveal a common CID fragment ion at $m / z 723.6$ $(-59 \mathrm{Da})$ corresponding to neutral loss of trimethylamine from the phosphocholine head-group [20, 45]. The peaks appearing at $\mathrm{m} / \mathrm{z} 672.5$ and $686.5 \mathrm{can}$ be confidently assigned to the ozone-induced neutral losses of -110 and $-94 \mathrm{Da}$, respectively, as expected from the n-9 fatty acyl chain present in each lipid. The inset to Figure $5 \mathrm{c}$ and $\mathrm{d}$ shows the mass range $\mathrm{m} / \mathrm{z}$ 650-700 with the vertical scale magnified by a factor of 30 highlighting the different abundance of the OzID product peaks at $\mathrm{m} / \mathrm{z} 672.5$ and 688.5. Integrating and normalizing the area under the peaks reveals that the $[\mathrm{PC}(16: 0 / 9 \mathrm{Z}-18: 1)+\mathrm{Na}]^{+}$regioisomer reacts some 2.8 times faster than $[\mathrm{PC}(9 \mathrm{Z}-18: 1 / 16: 0)+\mathrm{Na}]^{+}$. It should be noted that this value may represent an underestimate of the relative reactivity of these regioisomers as Ekroos et al. have previously demonstrated that even synthetic standards (such as those used here) can include up to $21 \%$ of the alternative regioisomer [6]. The increased reactivity of the unsaturated radyl at the $s n-2$ position suggests either greater accessibility of the olefin for reaction and/or a favorable interaction between the olefin and the charge, activating it toward ozonolysis.

\section{Identifying Isomeric Lipids in a Complex Extract}

Figure 6a shows the electrospray ionization mass spectrum obtained by direct infusion of a crude lipid extract from a cow brain. The presence of sodium acetate in the infusing solution leads to formation of $[\mathrm{M}+\mathrm{Na}]^{+}$for each molecular phospholipid with the positive-ion spectrum dominated by choline-containing lipids. The base peak at $m / z 782.6$ corresponds in mass to a sodiated phosphatidylcholine with a stoichiometry of PC(34:1). Figure $6 \mathrm{~b}$ shows a tandem mass spectrum obtained from mass-selected $\mathrm{m} / \mathrm{z} 782.6$ ions with ozone vapor present in q2 of the mass spectrometer. Instrumental parameters for this spectrum (including a collision energy $5 \mathrm{eV}$ and a supplemental voltage of $20 \mathrm{~V}$ ) were selected to facilitate both CID and OzID processes in a single acquisition. While seemingly complex, most of the ions can be assigned to one of the two processes or a combination of both. For example, the CID product ions at $m / z 723.5[\mathrm{M}+\mathrm{Na}-59]^{+}$and $599.6[\mathrm{M}+\mathrm{Na}-$ $183]^{+}$confirm the assignment of the phosphocholine head group while the secondary CID fragment ions at $\mathrm{m} / \mathrm{z} 441.3\left[\mathrm{M}+\mathrm{Na}-59-\mathrm{C}_{17} \mathrm{H}_{33} \mathrm{CO}_{2} \mathrm{H}\right]^{+}$and $467.4[\mathrm{M}+$ Na-59- $\left.\mathrm{C}_{15} \mathrm{H}_{31} \mathrm{CO}_{2} \mathrm{H}\right]^{+}$identify the number of carbons and degree of unsaturation of each of the acyl chains (i.e., 18:1 and 16:0, respectively). As discussed above, the abundance of the product ion arising from neutral loss of the $s n-1$ radyl is generally more abundant. In this spectrum, however, the relative abundance of these two product ions $\mathrm{m} / \mathrm{z} 441.3$ and 467.4 (labeled with circles) is close to parity, suggesting that both sn-positional isomers, namely $\mathrm{PC}(16: 0 / 18: 1)$ and $\mathrm{PC}(18: 1 / 16: 0)$, are present in the lipid extract.

Figure $6 \mathrm{~b}$ also shows a pair of OzID ions at $m / z 672.4$ and 688.4 (labeled with diamonds) corresponding to neutral losses of -110 and $-94 \mathrm{Da}$ and indicative of an $n-9$ double-bond. Interestingly, a second pair of ions is also observed in Figure 6b, some 28 Th higher in mass at $m / z 700.4$ and 716.4 (labeled with diamonds). These ions correspond to neutral losses -82 and $-66 \mathrm{Da}$ and are thus characteristic OzID product ions arising from a lipid ion containing an n-7 double-bond [31]. A further set of four OzID ions arising from oxidative dissociation of the $[\mathrm{M}+\mathrm{Na}-59]^{+}$ion are also observed and are labeled with an asterisk. Given that the mass-selected precursor ion is monounsaturated, the OzID ions in this spectrum clearly identify the presence of two isomeric phospholipids in the crude extract.

As discussed above, the relative abundance ratio of the OzID ions in Figure $6 \mathrm{~b}$ may give an indication of the stereochemistry of the double-bond(s). The abundance ratio of the aldehyde and Criegee ions arising from dissociation of the $n-9$ double-bond (i.e., $m / z 672.4$ and 688.4 , respectively) are present in a ratio of $\mathrm{A}_{672} / \mathrm{A}_{688} \sim$ 2.5 , which could be considered as a weighted average of the analogous ratios for the standards $\mathrm{PC}(16: 0 / 9 \mathrm{Z}-18: 1)$ $\left(\mathrm{A}_{672} / \mathrm{A}_{688} \sim 2.7\right)$ and PC $(9 \mathrm{Z}-18: 1 / 16: 0)\left(\mathrm{A}_{672} / \mathrm{A}_{688} \sim\right.$ 2.3) under the same experimental conditions (see Supplementary Material, Figure S6). The congruence observed in these ion abundances provides sufficient evidence for preliminary assignment of cis-stereochemistry to these brain-derived n-9 phosphatidylcholines. Confirmation of this assignment would require direct comparison with authentic samples of the $n-9$ transisomers $\mathrm{PC}(16: 0 / 9 E-18: 1)$ and $\mathrm{PC}(9 E-18: 1 / 16: 0)$ that were not available for this study. Furthermore, no 


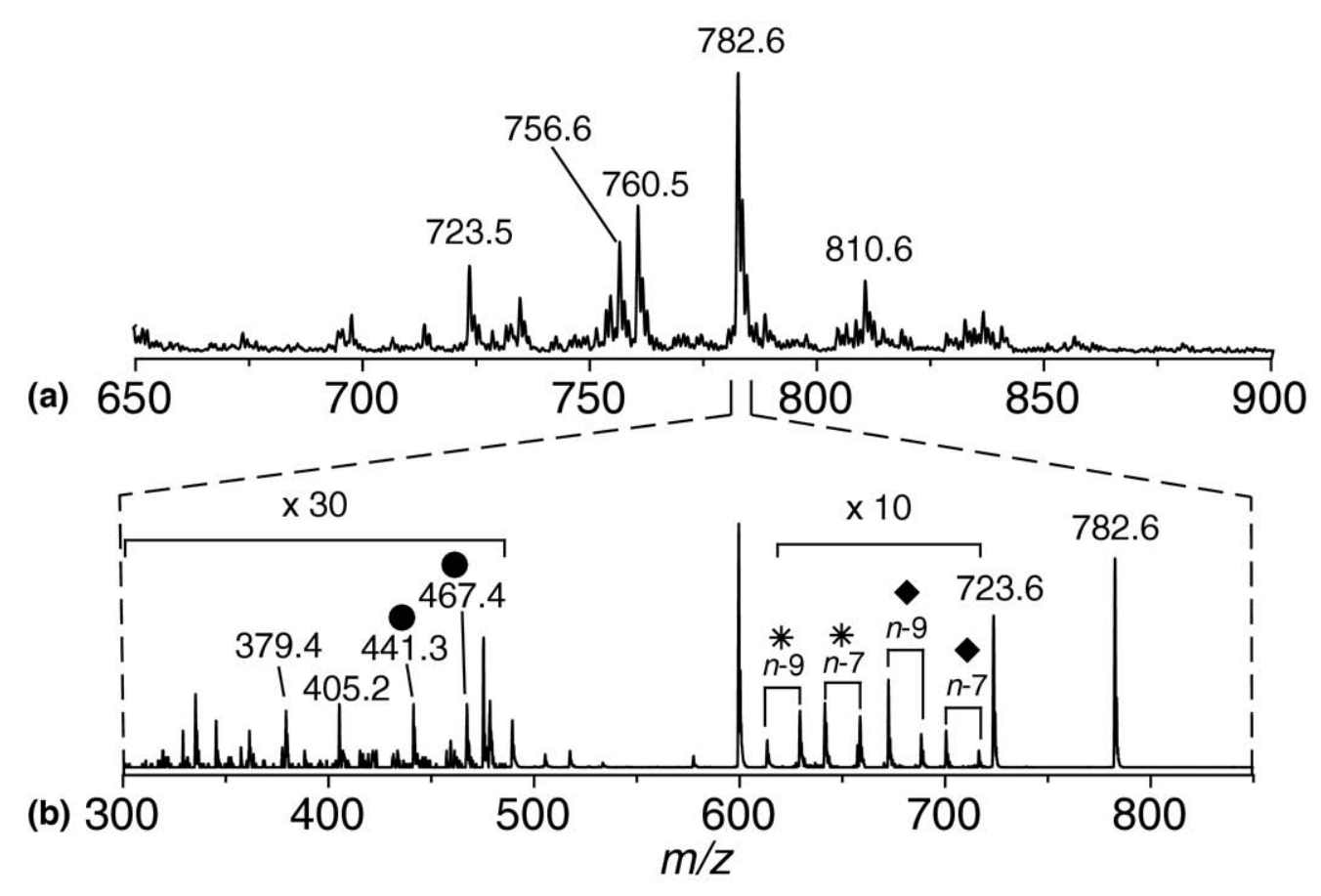

\section{(c)}

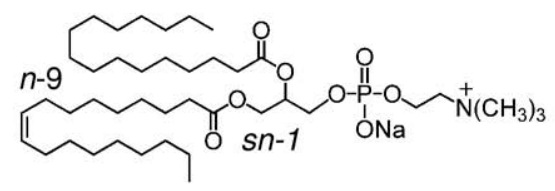

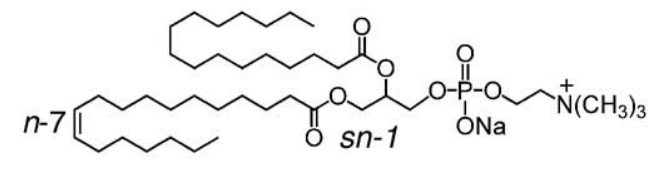

Figure 6. (a) ESI-MS spectrum obtained by direct infusion of a crude lipid extract from cow brain. (b) Combined CID-OzID spectrum acquired by applying collision energy to the mass-selected precursor ion at $\mathrm{m} / \mathrm{z} 782.6$ with ozone vapor present in the collision cell (q2). The labeling of the various ions is described in the text. (c) The molecular structure of four regioisomeric lipids that could give rise to the combined spectral features observed in (b).

inference as to the stereochemistry of the $n-7$ phospholipids could be drawn as neither cis nor trans standards could be obtained.

In summary, the information-rich combination CIDOzID spectrum shown in Figure 6b clearly identifies the presence of multiple isomeric phospholipids that differ in the position of the double-bond within the monounsaturated 18:1 radyl (n-7 and n-9) and the relative position of this substituent on the glycerol backbone (both $s n-1$ and $s n-2)$. This indicates that the ion population at $m / z 782$ (Figure 6a) arises from a mixture of at least two isomeric lipids, e.g., $\mathrm{PC}(16: 0 / 9 \mathrm{Z}-18: 1)$ and PC(11Z-18:1/16:0), but possibly an even more complex mixture including all four isomeric phosphatidylcholine structures shown in Figure 6c: with tentative assignment of cis-stereochemistry in all cases. Unequivocal identification of the presence of these isomeric forms by other methods would require extensive fractionation of the crude extract [4].

\section{Conclusions}

The data presented herein demonstrate that OzID can be performed routinely on a commercially available tandem linear ion-trap mass spectrometer with only relatively minor modifications. The spatial separation of the ozone reaction from the mass-analysis stage in this configuration provides significant enhancements in the speed and sensitivity of the analysis with interpretable spectra acquired on the sub-second timescale (cf. Figure $2 b$ ). The spectra shown here further demonstrate that OzID is applicable to a wide range of lipids, yielding easily interpretable spectra for fatty acids, triacylglycerols, and phospholipids, including several different head groups (further examples provided as Supplementary Material, Figures S1 and S4). Significantly, OzID can be used to identify the presence of isomeric lipids in complex mixtures without prior fractionation. Furthermore the predictable nature of the ozone-induced frag- 
mentation allows for unambiguous assignment of double-bond position(s) and is applicable to de novo structure elucidation without the need for reference standards. Significantly, the observation that doublebond- and sn-positional isomerism give rise to different rates of reaction with ozone suggests caution in interpreting OzID ion abundances quantitatively. For example, the greater abundance of $n-9$ to $n-7$ product ions in Figure $6 \mathrm{~b}$ cannot be taken as evidence of a greater relative concentration of the $n-9$ lipids in this mixture.

The stable ozone concentration afforded by this instrumental configuration has provided the first opportunity to compare the relative reactivity of isomeric lipid ions toward ozone. The observation that both the rate and the branching ratio of the ozonolysis reaction are affected by the stereochemistry of the olefin suggests possible application of OzID in differentiating of cis and trans double bonds. Differences in the spectra of stereoisomers however, lie only in the relative abundance of OzID ions rather than in any unique products and thus-without a more detailed understanding of the other structural and mechanistic influences on the spectra-comparison with an authentic standard is essential. Furthermore, the reliance on abundance ratios, suggest a limited ability to characterize a mixture of stereoisomers.

Observations of differences in the OzID spectra of isomeric lipids differing in double-bond position and/or sn-position suggest that the rate of reaction with ozone can be influenced by these structural variations. This is intriguing, as the covalent bonding environment of the double-bond itself is essentially indistinguishable in such cases. As such, the evidence points to the influence of other factors, such as the three-dimensional structure of the ionized lipid in the gas-phase. This could influence the accessibility of the double-bond to incident ozone and/or the proximity of this moiety to other functional groups, particularly the charge carrier, may serve to activate it toward reaction with ozone. While further studies are required to characterize these influences carefully, there is sufficient evidence to suggest that quantification of isomeric lipids by OzID will require calibration.

The instrumental configuration described here has the demonstrated capability of acquiring both CID and OzID product ions in the same mass spectrum. This approach further increases the speed and sensitivity of the analysis and represents another step toward the goal of complete structural elucidation of lipids by mass spectrometry alone (that could be dubbed "top-down lipidomics"). The observation of up to four structurally distinct lipid isomers in the ion population of a single mass-to-charge ratio from an extract serves to highlight the potential of contemporary analytical methods to significantly underestimate the diversity of biologically derived lipids. Such observations also raise intriguing questions regarding the specific biochemical roles of these distinct but structurally related compounds.

\section{Acknowledgments}

The authors acknowledge AB SCIEX for provision of the 2000 QTRAP instrument with which these experiments were conducted, and Drs. Jim Hager and Yves Le Blanc for helpful suggestions. B.L.J.P. and H.T.P. were supported by the funding from the Australian Research Council (DP0986738 and DP0986628) and the University of Wollongong. Additional support was provided by Zora Biosciences. M.C.T. and J.R.N. were supported by Australian Postgraduate Awards.

\section{Appendix A Supplementary Material}

Supplementary material associated with this article may be found in the online version at doi:10.1016/ j.jasms.2010.08.011.

\section{References}

1. Vance, D. E.; Vance, J. E. Biochemistry of Lipids, Lipoproteins, and Membranes, 5th ed.; Elsevier: Amsterdam, 2008; p. 35.

2. Gurr, M. I.; Harwood, J. L.; Frayn, K. N. Lipid Biochemistry, 5th ed.; Blackwell Science Ltd.: Oxford, UK, 2002; p. 93.

3. Mossoba, M. M.; Kramer, J. K. G.; Brenna, J. T.; McDonald, R. E. Lipid Analysis and Lipidomics: New Techniques and Applications; AOCS Press: Champaign, IL, 2006; p. 5.

4. Mitchell, T. W.; Pham, H.; Thomas, M. C.; Blanksby, S. J. Identification of Double Bond Position in Lipids: From GC to OzID. J. Chromatogr. B 2009, 877, 2722-2735.

5. Menzel, D.; Olcott, H. Positional Distribution of Fatty Acids in Fish and Other Animal Lecithins. Biochim. Biophys. Acta 1964, 84, 133-139.

6. Ekroos, K.; Ejsing, C. S.; Bahr, U.; Karas, M.; Simons, K.; Shevchenko, A. Charting Molecular Composition of Phosphatidylcholines by Fatty Acid Scanning and Ion Trap $\mathrm{MS}^{3}$ fragmentation. J. Lipid Res. 2003, 44, 2181-2192.

7. Leskinen, H.; Suomela, J. P.; Pinta, J.; Kallio, H. Regioisomeric Structure Determination of $\alpha$ - and $\gamma$-Linolenoyldilinoleoylglycerol in Black Currant Seed Oil by Silver Ion High-Performance Liquid Chromatography and Mass Spectrometry. Anal. Chem. 2008, 80, 5788-5793.

8. Leskinen, H. M.; Suomela, J. P.; Kallio, H. P. Quantification of Triacylglycerol Regioisomers by Ultra-High-Performance Liquid Chromatography and Ammonia Negative Ion Atmospheric Pressure Chemical Ionization Tandem Mass Spectrometry. Rapid Commun. Mass Spectrom. 2010, 24, 1-5

9. Nakanishi, H.; Iida, Y.; Shimizu, T.; Taguchi, R. Separation and Quantification of $s n-1$ and $s n-2$ Fatty Acid Positional Isomers in Phosphatidylcholine by RPLC-ESIMS/MS. J. Biochem. (Tokyo) 2010, 147, 245-256.

10. Tyburczy, C.; Major, C.; Lock, A.; Destaillats, F.; Lawrence, P.; Brenna, J.; Salter, A.; Bauman, D. Individual Trans Octadecenoic Acids and Partially Hydrogenated Vegetable Oil Differentially Affect Hepatic Lipid and Lipoprotein Metabolism in Golden Syrian Hamsters. J. Nutr. 2009, 139, 257-263.

11. Mozaffarian, D. Trans Fatty Acids-Effects on Systemic Inflammation and Endothelial Function. Atherosclerosis 2006, 7(Suppl.), 29-32.

12. Han, X.; Gross, R. W. Global Analyses of Cellular Lipidomes Directly from Crude Biological Samples by ESI Mass Spectrometry: A Bridge to Lipidomics. J. Lipid Res. 2003, 44, 1071-1079.

13. Fahy, E.; Subramaniam, S.; Brown, H. A.; Glass, C. K.; Merrill, A. H. Jr.; Murphy, R. C.; Raetz, C. R. H.; Russell, D. W.; Seyama, Y.; Shaw, W.; Shimizu, T.; Spener, F.; van Meer, G.; VanNieuwenhze, M. S.; White, S. H.; Witztum, J. L.; Dennis, E. A. A Comprehensive Classification System for Lipids. J. Lipid Res. 2005, 46, 839-861.

14. Ejsing, C. S.; Sampaio, J. L.; Surendranath, V.; Duchoslav, E.; Ekroos, K.; Klemm, R. W.; Simons, K.; Shevchenko, A. Global Analysis of the Yeast Lipidome by Quantitative Shotgun Mass Spectrometry. Proc. Natl. Acad. Sci. U.S.A. 2009, 106, 2136-2141.

15. Pulfer, M.; Murphy, R. C. Electrospray Mass Spectrometry of Phospholipids. Mass Spectrom. Rev. 2003, 22, 332-364.

16. Hsu, F. F.; Turk, J. Electrospray Ionization with Low-Energy Collisionally Activated Dissociation Tandem Mass Spectrometry of Glycerophospholipids: Mechanisms of Fragmentation and Structural Characterization. J. Chromatogr. B 2009, 877, 2673-2695.

17. Han, X.; Gross, R. W. Shotgun Lipidomics: Electrospray Ionization Mass Spectrometric Analysis and Quantitation of Cellular Lipidomes Directly from Crude Extracts of Biological Samples. Mass Spectrom. Rev. 2005, 24 , 367-412.

18. Han, X. Lipidomics: Developments and Applications. J. Chromatogr. B 2009, 877, 2663

19. Hsu, F. F.; Turk, J. Structural Characterization of Triacylglycerols as Lithiated Adducts by Electrospray Ionization Mass Spectrometry Using 
Low-Energy Collisionally Activated Dissociation on a Triple Stage Quadrupole Instrument. J. Am. Soc. Mass Spectrom. 1999, 10, 587-599.

20. Hsu, F.; Turk, J. Electrospray Ionization/Tandem Quadrupole Mass Spectrometric Studies on Phosphatidylcholines: The Fragmentation Processes. J. Am. Soc. Mass Spectrom. 2003, 14, 352-363.

21. Moe, M. K.; Anderssen, T.; Strom, M. B.; Jensen, E. Total Structure Characterization of Unsaturated Acidic Phospholipids Provided by Vicinal Dihydroxylation of Fatty Acid Double Bonds and Negative Electrospray Ionization Mass Spectrometry. J. Am. Soc. Mass Spectrom. 2005, 16, 46-59.

22. Thomas, M. C.; Mitchell, T. W.; Harman, D. G.; Deeley, J. M.; Murphy, R. C.; Blanksby, S. J. Elucidation of Double Bond Position in Unsaturated Lipids by Ozone Electrospray Ionization Mass Spectrometry. Anal. Chem. 2007, 79, 5013-5022.

23. Murphy, R. C.; Fiedler, J.; Hevko, J. Analysis of Nonvolatile Lipids by Mass Spectrometry. Chem. Rev. 2001, 101, 479-526.

24. Griffiths, W. J. Tandem Mass Spectrometry in the Study of Fatty Acids, Bile Acids, and Steroids. Mass Spectrom. Rev. 2003, 22, 81-152.

25. Cheng, C. F.; Gross, M. L. Complete Structural Elucidation of Triacylglycerols by Tandem Sector Mass Spectrometry. Anal. Chem. 1998, 70, 4417-4426.

26. Pittenauer, E.; Allmaier, G. The Renaissance of High-Energy CID for Structural Elucidation of Complex Lipids: MALDI-TOF/RTOF-MS of Alkali Cationized Triacylglycerols. J. Am. Soc. Mass Spectrom. 2009, 20, 1037-1047.

27. Hsu, F. F.; Turk, J. Structural Characterization of Unsaturated Glycerophospholipids by Multiple-Stage Linear Ion-Trap Mass Spectrometry with Electrospray Ionization. J. Am. Soc. Mass Spectrom. 2008, 19, 1681-1691.

28. Hsu, F. F.; Turk, J. Electrospray Ionization Multiple-Stage Linear Ion-trap Mass Spectrometry for Structural Elucidation of Triacylglycerols: Assignment of Fatty Acyl Groups on the Glycerol Backbone and Location of Double Bonds. J. Am. Soc. Mass Spectrom. 2010, 21, 657-669.

29. Thomas, M.; Mitchell, T.; Harman, D.; Deeley, J.; Nealon, J.; Blanksby, S. Ozone-Induced Dissociation: Elucidation of Double Bond Position Within Mass-Selected Lipid Ions. Anal. Chem. 2008, 80, 303-311.

30. Deeley, J. M.; Thomas, M. C.; Truscott, R. J. W.; Mitchell, T. W.; Blanksby, S. J. Identification of Abundant Alkyl Ether Glycerophospholipids in the Human Lens by Tandem Mass Spectrometry Techniques. Anal. Chem. 2009, 81, 1920-1930.

31. Thomas, M. C.; Mitchell, T. W.; Blanksby, S. J. On-Line Ozonolysis Methods for the Determination of Double Bond Position in Unsaturated Lipids. In Lipidomics, Armstrong, D., Eds.; Humana Press: Buffalo, 2009; p. $413-442$

32. Hager, J. A New Linear Ion Trap Mass Spectrometer. Rapid Commun. Mass Spectrom. 2002, 16, 512-526.
33. Nealon, J. R.; Blanksby, S. J.; Mitchell, T. W.; Else, P. L. Systematic Differences in Membrane Acyl Composition Associated with Varying Body Mass in Mammals Occur in All Phospholipid Classes: An Analysis of Kidney and Brain. J. Exp. Biol. 2008, 211, 3195-3204.

34. Fahy, E.; Subramaniam, S.; Murphy, R. C.; Nishijima, M.; Raetz, C. R. H.; Shimizu, T.; Spener, F.; van Meer, G.; Wakelam, M. J. O.; Dennis, E. A. Update of the LIPID MAPS Comprehensive Classification System for Lipids. J. Lipid Res. 2009, 50, S9-S14.

35. Mansoori, B.; Dyer, E.; Lock, C.; Bateman, K.; Boyd, R.; Thomson, B. Analytical Performance of a High-Pressure Radio Frequency-Only Quadrupole Collision Cell with an Axial Field Applied by Using Conical Rods. J. Am. Soc. Mass Spectrom. 1998, 9, 775-788.

36. Londry, F. A.; Hager, J. W. Mass Selective Axial Ion Ejection from a Linear Quadrupole Ion Trap. J. Am. Soc. Mass Spectrom. 2003, 14, 1130-1147.

37. Hager, J. Product Ion Spectral Simplification Using Time-Delayed Fragment Ion Capture with Tandem Linear Ion Traps. Rapid Commun. Mass Spectrom. 2003, 17, 1389-1398.

38. IDLH Documentation-Centers for Disease Control and Prevention. http://www.cdc.gov/Niosh/idlh/10028156.html; accessed 12 February 2010.

39. Criegee, R. Mechanism of Ozonolysis. Angew. Chem. Int. Ed. 1975, 14, 745-752.

40. Taatjes, C. A.; Meloni, G.; Selby, T. M.; Trevitt, A. J.; Osborn, D. L.; Percival, C. J.; Shallcross, D. E. Direct Observation of the Gas-Phase Criegee Intermediate $\left(\mathrm{CH}_{2} \mathrm{OO}\right)$. J. Am. Chem. Soc. 2008, 130, 1188311885.

41. Taylor, I. F.; Blanksby, S. J.; Colbran, S. B.; Willet, G. D. Production and Isolation of Ligated Metal(IV)-Oxo Ions by Tandem Mass Spectrometry. Rapid Commun. Mass Spectrom. 2010, 24, 1142-1146.

42. Williams, S.; Campos, M.; Midey, A.; Arnold, S.; Morris, R.; Viggiano, A Negative Ion Chemistry of Ozone in the Gas Phase. J. Phys. Chem. A 2002, 106, 997-1003.

43. Grosjean, E.; Grosjean, D. Rate Constants for the Gas-Phase Reaction of Ozone with Unsaturated Oxygenates. Int. J. Chem. Kinet. 1998, 30, 21-29.

44. Grosjean, E.; Grosjean, D. The Reaction of Unsaturated Aliphatic Oxygenates with Ozone. J. Atmos. Chem. 1999, 32, 205-232.

45. Domingues, P.; Domingues, M. R.; Amado, F. M. L.; Correia, A. J. F. Characterization of Sodiated Glycerol Phosphatidylcholine Phospholipids by Mass Spectrometry. Rapid Commun. Mass Spectrom. 2001, 15, 799-804.

46. Greene, C. R.; Atkinson, R. Rate Constants for the Gas Phase Reactions of $\mathrm{O}_{3}$ with a Series of Alkenes at $296 \mathrm{~K} \pm 2 \mathrm{~K}$. Int. J. Chem. Kinet. 1992, 24, 803-811.

47. Vayner, G.; Addepalli, S. V.; Song, K.; Hase, W. L. Post-Transition State Dynamics for Propene Ozonolysis: Intramolecular and Unimolecular Dynamics of Molozonide. J. Chem. Phys. 2006, 125, 014317. 